\title{
Prediction of early outcome in resolving chronic lung disease of prematurity after discharge from hospital
}

\author{
R Iles, A T Edmunds
}

\begin{abstract}
In an attempt to identify those infants with resolving chronic lung disease of prematurity (CLD) at greatest risk of sudden infant death syndrome or acute life threatening event (SIDS/ALTE), or readmission to hospital following discharge, recordings of arterial oxygen saturation were made on 35 infants. Recordings were collected while the infants were breathing room air. Movement artefact was excluded and the data analysed to provide the mean individual arterial oxygen saturation $\left(\mathrm{MSaO}_{2}\right)$, and the variability of the mean individual oxygen saturation $\left(\delta \mathrm{MSaO}_{2}\right)$. These data were related to clinical outcome recorded over the three months following investigation. A $\mathrm{MSaO}_{2}$ less than $90 \%$ on discharge predicted hospital admission within three months with a sensitivity of 1 and a specificity of $0 \cdot 76$, and SIDS/ALTE with a sensitivity of 1 and a specificity of 0.75 . A $\delta \mathrm{MSaO}_{2}$ greater than $6 \%$ predicted SIDS/ALTE with a sensitivity 0.88 and specificity of 1 . Infants with resolving chronic lung disease of prematurity who are at risk of increased morbidity and mortality can be assessed by accurate measurement of mean arterial saturation.

(Arch Dis Child 1996; 74: 304-308)
\end{abstract}

Keywords: bronchopulmonary dysplasia, outcome, sudden infant death syndrome, oximetry.

The Royal Hospital for Sick Children, Sciennes Road, Edinburgh EH9 1LF $R$ Iles A T Edmunds

Correspondence to: Dr R Iles, Department of Respiratory Medicine and Research, The Hospital for Sick Children, 555 University Avenue, Toronto Ontario, Canada, M5G 1 X8.

Accepted 16 November 1995 hypoxaemia with or without apnoea. This is particularly true of the infant with resolving lung disease, who is ready for discharge from a neonatal unit. The decision whether to prescribe supplementary oxygen is often based on clinical criteria rather than physiological assessment. Many infants no longer have a clinical requirement for supplementary oxygen, but still have unresolved lung disease. They are prone to hypoxaemia, particularly at times when they are stressed by intercurrent respiratory infection.

In a prospective study we aimed to test the hypothesis that recordings of mean individual arterial oxygen saturation and its variability could be used to assess the risk of morbidity and mortality in infants with significant lung disease of prematurity. The provision of appropriate support services such as home oxygen, home apnoea monitoring, and practical instruction in resuscitation could then be targeted more effectively.

\section{Methods}

Infants with CLD were recruited from the regional neonatal unit, the Simpson Memorial Maternity Pavilion, Edinburgh. This unit serves a defined geographical area of the Lothian Region of South East Scotland. Forty eight infants who had been born prematurely were identified as being eligible to enter the study when they had required 28 days of continuous supplementary oxygen from birth. The parents of these infants were provided with both verbal and written information at least one month before being asked to give consent for their infants to take part in the study. Forty of the 48 infants $(83 \%)$ were recruited. Three infants were initially transferred from outside the Lothian region. The care of these infants was returned to the referring hospitals and they were not included in this prospective study. The parents of five infants did not give consent to the study.

Thirty seven infants were studied before discharge from the neonatal unit. Three infants were not discharged and were studied when clinically stable. Monitoring errors and computer malfunction resulted in insufficient data collection for further analysis in five infants.

The study was approved by the paediatrics/reproductive medicine research ethics subcommittee of the Lothian research ethics committee.

\section{MONITORING OF ARTERIAL OXYGEN} SATURATION

All monitoring was done on an inpatient basis. Real time values of heart rate were taken from the analogue port of a Kontron Minimon 7135 
or the serial port of a Hewlett Packard 78834A monitor. Real time values for heart rate and oxygen saturation were taken from the serial output of an Ohmeda Biox 3700 pulse oximeter. Infants were monitored for at least a 6 hour period overnight. The pulse oximeter probe was attached initially on the lateral aspect of the foot, but moved to the lateral aspect of the hand if a poor signal was obtained. The probe was fixed with adhesive spots as supplied by Ohmeda and was then wrapped lightly with a Coban tape bandage. Data was collected, stored in real time, and analysed by the MARY patient monitoring system (Meadowbank Medical Systems). ${ }^{7}$ Input data were collected and stored at a rate of $1 \mathrm{~Hz}$. The mean value for each minute was used as the analysed data point. Verification of the use of the mean value for each minute was made by separate analysis of two 1 hour periods of collected data on each patient, using the data collected at $1 \mathrm{~Hz}$.

Artefacts resulting from movement or probe disconnection were excluded initially by visual inspection of the trace and then reanalysed more specifically by comparing the heart rate obtained from the heart rate monitor to that from the Ohmeda pulse oximeter. Where there was more than a $5 \%$ difference, the $\mathrm{SaO}_{2}$ data were discarded as being influenced by artefact.

Analysis of data collected over at least a 6 hour period after the exclusion of artefact gave for each infant the mean arterial oxygen saturation $\left(\mathrm{MSaO}_{2}\right)$ and the variability of the mean arterial oxygen saturation $\left(\delta \mathrm{MSaO}_{2}\right)$ (defined as $\pm 1 \mathrm{SD}$ ).

In the six infants requiring continuous oxygen, oxygen was discontinued to obtain steady state values for $\mathrm{SaO}_{2}$ while breathing air. This was done with informed parental consent, when the infant was asleep, or settled after a feed. The infants were monitored by both ECG and pulse oximetry. Oxygen was then restarted if the infant's arterial oxygen saturation was constantly below $90 \%$ in air. For these infants values for $\mathrm{MSaO}_{2}$ and $\delta \mathrm{MSaO}_{2}$ were obtained from the time that the infants were breathing air alone. In three infants oxygen saturation fell rapidly below $80 \%$ in air. Oxygen was restarted immediately after the lowest 'spot value' of $\mathrm{SaO}_{2}$ had been recorded. This was taken as the $\mathrm{MSaO}_{2}$ for the infant in subsequent analysis. No value for $\delta \mathrm{MSaO}_{2}$ was recorded in these infants. Oxygen flow was then titrated to give $\mathrm{SaO}_{2}$ values of $92-93 \%$. Infants were closely monitored throughout this procedure. No infant became bradycardic or apnoeic. Data were analysed to give values for $\mathrm{MSaO}_{2}$ on 35 infants and for $\delta \mathrm{MSaO}_{2}$ in 32 .

Decisions regarding an infant's suitability for discharge with or without the prescription of home oxygen were made by clinicians not involved in the study. They were based on the overall clinical perception of the infant and visual analysis of the same periods of oxygen saturation data as were analysed. Those infants who were judged not to require supplementary oxygen on clinical grounds were not counselled regarding SIDS/ALTE.
CLINICAL OUTCOME

The respiratory unit of the Royal Hospital for Sick Children was notified by the parents of infants who were admitted to hospital during the three month study period. Those admitted were screened for respiratory pathogens by bacterial and viral culture of throat swab and nasopharyngeal secretions. Respiratory syncitial virus (RSV) was specifically screened for by rapid direct fluorescence, and then by tissue culture using skin fibroblast cells or monkey kidney cells or both.

An apparent life threatening event was defined as 'an episode that was frightening to the observer and was characterised by some combination of central apnoea, colour change with marked change in muscle tone' ${ }^{8}$ The diagnosis of an ALTE was made from the detailed history of the event recorded by an independent clinician.

\section{STATISTICAL ANALYSIS}

Data were analysed to give the mean and 95 percent confidence intervals for the cohort, and for the infants not receiving oxygen. The unpaired Student $t$ test was used to test the null hypothesis that hospital admission or SIDS/ALTE was independent of measurements of $\mathrm{MSaO}_{2}$ and $\delta \mathrm{MSaO}_{2}$. Probability (p) values less than 0.05 were considered statistically significant.

Receiver operating characteristic (ROC) plots compared the performance of the method of exclusion of artefact. ${ }^{9}$ They were created by plotting sensitivity against 1 -specificity for $\mathrm{MSaO}_{2}$ and $\delta \mathrm{MSaO}_{2}$, for prediction of hospital admission and SIDS/ALTE, collected before and after the exclusion of artefact.

\section{Results}

Forty infants were recruited. They were born between 24 and 31 weeks of gestation (median 27 weeks). They weighed a median of $900 \mathrm{~g}$ (range 589-1891 g) at birth. All infants required ventilatory support within the first week after birth. This was required for a median of 25 days (range 6-120 days). Thirty three of the infants had required supplemental oxygen at 36 weeks postconceptional age. The range of corrected gestational age at time of study was $39-42$ weeks.

The clinical course of the five infants who were eligible for, but not included in, the study as their parents did not give consent, was recorded. Three infants were readmitted to hospital with respiratory tract infection. One was admitted following an ALTE. No evidence of infection was recorded on this infant. One infant was lost to follow up. These data have not been included in the analysis of the cohort studied as no physiological data were available.

At the time of study six infants were receiving supplementary oxygen. Three of these had evidence of right ventricular hypertrophy on ECG and had raised carbon dioxide tensions from capillary blood gases. All other infants had normal ECGs and capillary blood gases at the time of study. 
Sensitivity and specificity of oxygen saturation measurements in predicting hospital readmission and SIDS/ALTE

\begin{tabular}{|c|c|c|c|c|c|}
\hline & & \multicolumn{2}{|c|}{ Hospital readmission } & \multicolumn{2}{|c|}{$S I D S / A L T E$} \\
\hline & & Sensitivity & Specificity & Sensitivity & Specificity \\
\hline & $\begin{array}{l}\mathrm{MSaO}_{2}<90 \% \\
\mathrm{MSaO}_{2}<90 \% \\
\delta \mathrm{MSaO}_{2}>6 \% \\
8 \mathrm{MSaO}_{2}>6 \%\end{array}$ & $\begin{array}{l}0.8 \\
1 \\
0.05 \\
0.52\end{array}$ & $\begin{array}{l}0 \cdot 73 \\
0 \cdot 76 \\
0 \cdot 86 \\
0 \cdot 13\end{array}$ & $\begin{array}{l}1 \\
1 \\
0 \\
0 \cdot 88\end{array}$ & $\begin{array}{l}0 \cdot 57 \\
0 \cdot 75 \\
0 \cdot 84 \\
1\end{array}$ \\
\hline
\end{tabular}

(A) Data without exclusion of artefact; (B) data following exclusion of artefact. SIDS = sudden infant death syndrome; $\mathrm{ALTE}=$ apparent life threatening event; $\mathrm{MSaO}_{2}=$ mean arterial oxygen saturation; $\delta \mathrm{MSaO}_{2}=$ change in mean arterial oxygen saturation.

Of the cohort studied, 20 of the 40 infants were readmitted to hospital $(50 \%)$. Five of the six infants who received oxygen $(83 \%)$, and 15 of the 34 of those that did not $(44 \%)$, were readmitted. On hospital admission, 11 of the infants had proven RSV infection, one parainfluenza, and one rhinovirus infection. Eight infants were admitted with clinical history of ALTE. Infection was not proven in these infants. One infant died unexpectedly. The necropsy was performed by a paediatric pathologist aware of the child's neonatal history. The cause of death was cited as SIDS. The changes of chronic lung disease were noted to be minimal and felt not to be a significant factor in the child's death. This child was not receiving supplementary oxygen at the time of death.

The mean period of recorded data for the 29 infants who had been identified on clinical grounds as not having a requirement for supplementary oxygen at time of study was 9.5 hours (6-12 hours). Mean percentage time

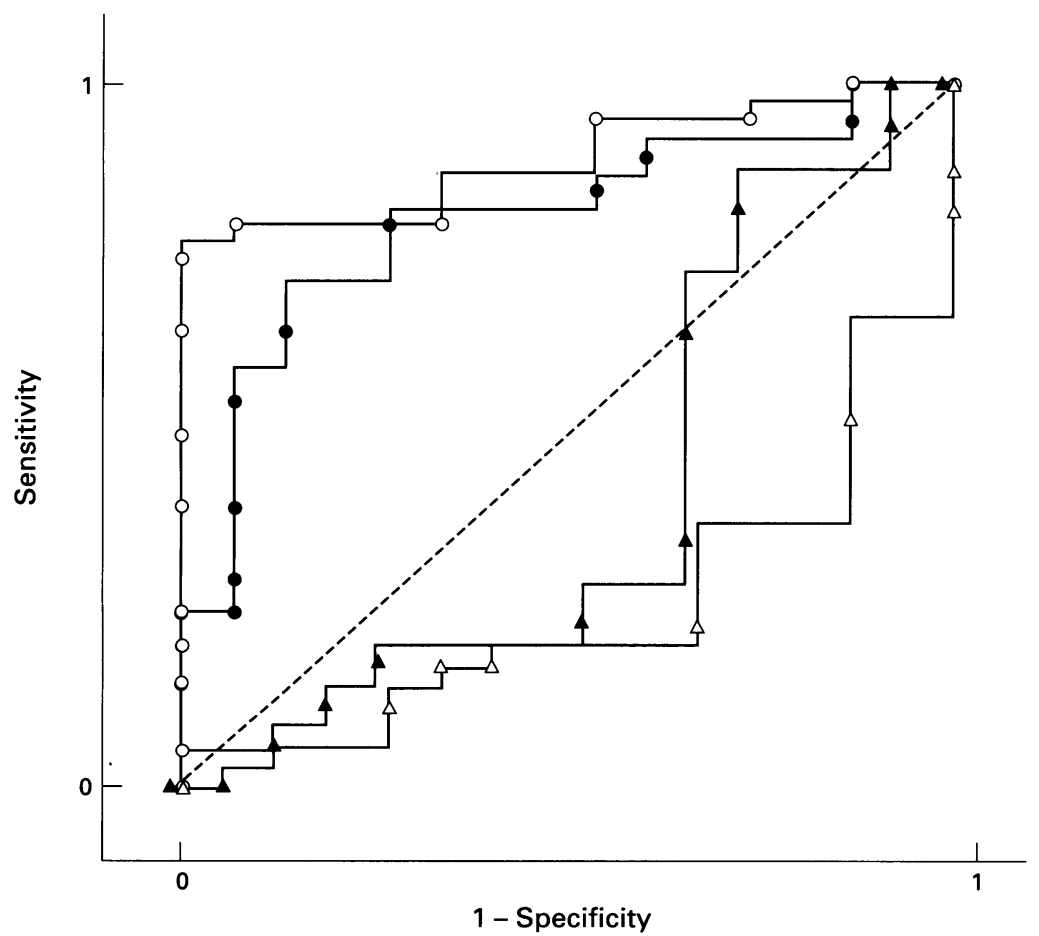

$$
\begin{aligned}
& \longrightarrow \text { Prediction of readmission by } \mathrm{MSaO}_{2} \\
& \longrightarrow-\text { Prediction of readmission by } \mathrm{MSaO}_{2} \text { (artefact removed) } \\
& \rightarrow-\text { Prediction of readmission by } \delta \mathrm{MSaO}_{2} \\
& \rightarrow-\text { Prediction of readmission by } \delta \mathrm{MSaO}_{2} \text { (artefact removed) }
\end{aligned}
$$

Figure 1 ROC curves for the prediction of hospital readmission, by $\mathrm{MS}_{\mathrm{OO}} \mathrm{O}_{2}$ and $\mathrm{\delta MSaO} \mathrm{O}_{2}$. excluded due to artefact was $38 \cdot 2 \%$ (range $3 \%$ to $81 \%$ ) of total time.

The range of $\mathrm{MSaO}_{2}$ for those infants who had been identified on clinical grounds as not having a requirement for supplementary oxygen was $86.5 \%$ to $96.4 \%$. The cohort $\mathrm{MSaO}_{2}$ varied between $75 \%$ and $96.8 \%$ in air. Nine of the 34 infants (31\%) discharged without supplemental oxygen had $\mathrm{MSaO}_{2}$ values less than $90 \%$ after the exclusion of artefact.

For data analysed with and without the exclusion of artefact, the sensitivity and specificity of a $\mathrm{MSaO}_{2}$ less than $90 \%$ and $\delta \mathrm{MSaO}_{2}$ greater than $6 \%$ to predict hospital admission and SIDS/ALTE is shown in the table. The exclusion of artefact improved the performance of an $\mathrm{MSaO}_{2}$ value of less than $90 \%$ in predicting hospital readmission by increasing the sensitivity from 0.8 to 1 and specificity from $0 \cdot 73$ to $0 \cdot 76$. The sensitivity of prediction of SIDS/ALTE by $\mathrm{MSaO}_{2}$ was improved from 0.57 to 0.75 . Similarly the performance a $\delta \mathrm{MSaO}_{2}$ greater than $6 \%$ to predict SIDS/ ALTE was increased from a sensitivity from 0 to 0.88 and from specificity 0.84 to 1 . The exclusion of artefact improved the prediction of hospital readmission by $\mathrm{MSaO}_{2}$, but its prediction by $\delta \mathrm{MSaO}_{2}$ remained uninformative (fig 1). It improved the prediction of SIDS/ALTE by $\mathrm{MSaO}_{2}$ and its prediction by $\delta \mathrm{MSaO}_{2}$ from a random assessment to the most discriminating of the tests (fig 2).

There was a significant difference between the group means for $\mathrm{MSaO}_{2}$ (fig 3) and $\delta \mathrm{MSaO}_{2}$ (fig 4) when data from those infants readmitted to hospital were compared to those not admitted. There was also a significant difference between the group means for those infants who suffered an acute life threatening event or died of sudden infant death syndrome when compared with those who did not. These differences remained significant when data from only those infants who did not receive supplemental oxygen on discharge were compared.

\section{Discussion}

Using a method of analysis that excludes artefact from recordings of arterial oxygen saturation, we have been able to calculate accurate values for mean individual arterial saturation and its variability and to improve their predictive accuracy. Using these data we have identified a limit of $90 \%$ for mean arterial saturation below which infants with resolving chronic lung disease are at increased risk of sudden infant death syndrome, an acute life threatening event, or hospital admission. Similarly a limit of $6 \%$ variability was identified above which infants were at increased risk of sudden infant death or an acute life threatening event.

There is no clear consensus on the prescription of supplemental oxygen to infants with chronic lung disease. Ours are the only published data that support the practice of many neonatal units in prescribing oxygen for infants who have a mean arterial saturation in air of less than $90 \%$. Historically this practice has been based on data and recommendations 
from adult studies. Administration of low flow oxygen to adults with chronic obstructive pulmonary disease whose $\mathrm{PaO}_{2}$ is less than $7 \cdot 8 \mathrm{kPa}$ $(60 \mathrm{~mm} \mathrm{Hg}$ ) prevents the progression of their pulmonary hypertension and decreases mortality. ${ }^{1011}$ In infants with severe pulmonary disease, pulmonary hypertension is responsive
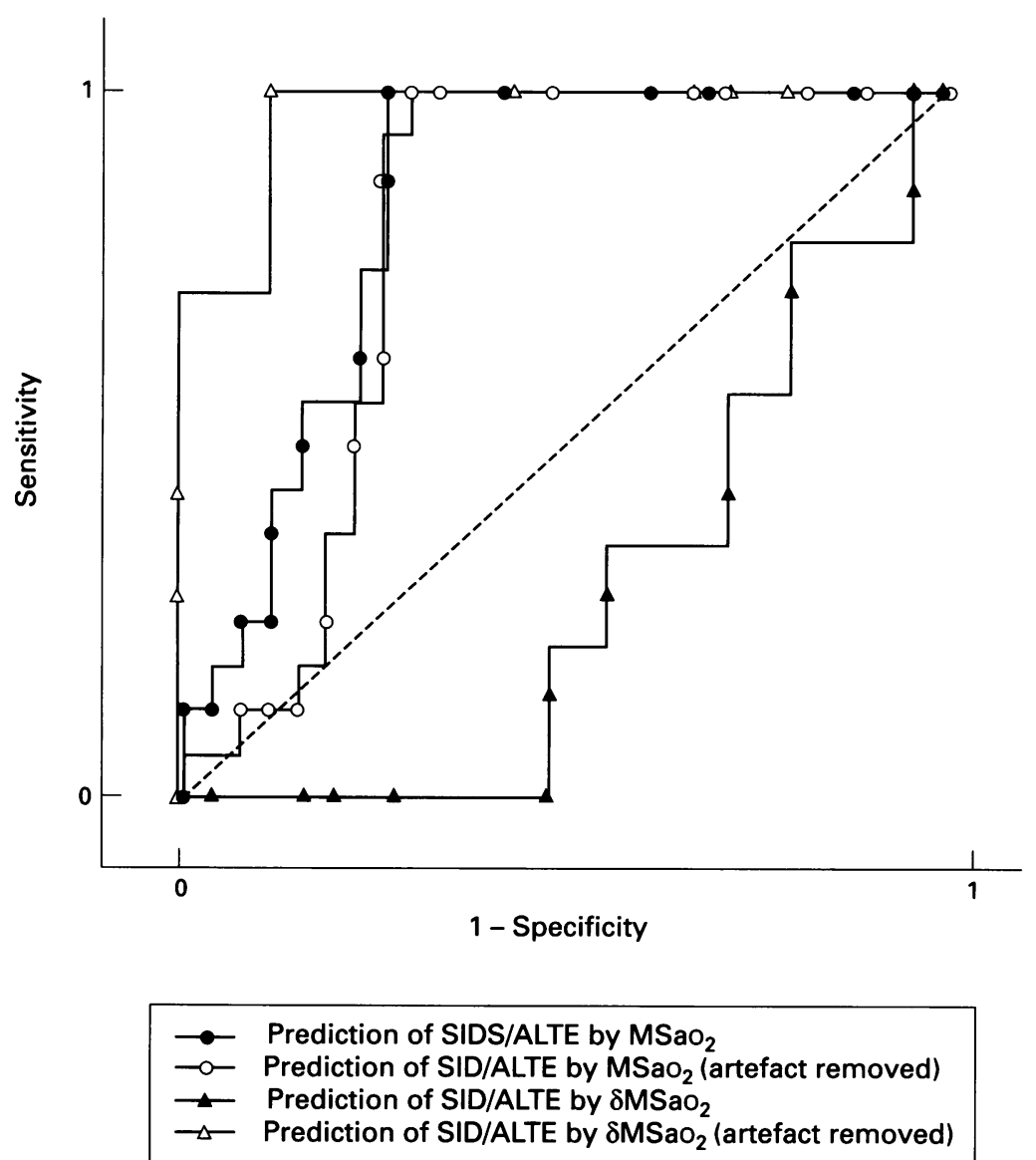

Figure 2 ROC curves for prediction of SIDS/ALTE by $\mathrm{MSaO}_{2}$ and $\delta \mathrm{MSaO}_{2}$.

Figure $3 \quad \mathrm{MSaO}_{2}$ and $95 \%$ confidence intervals for clinical outcome groups. SIDS/ALTE v no SIDS/no ALTE: whole cohort, $p=0.04$; infants not receiving supplemental oxygen, $p=0.0001$.

Hospital readmission $\mathrm{v}$ no hospital readmission: whole cohort, $p=0 \cdot 001$; infants not receiving supplemental oxygen, $p=0.0002$.
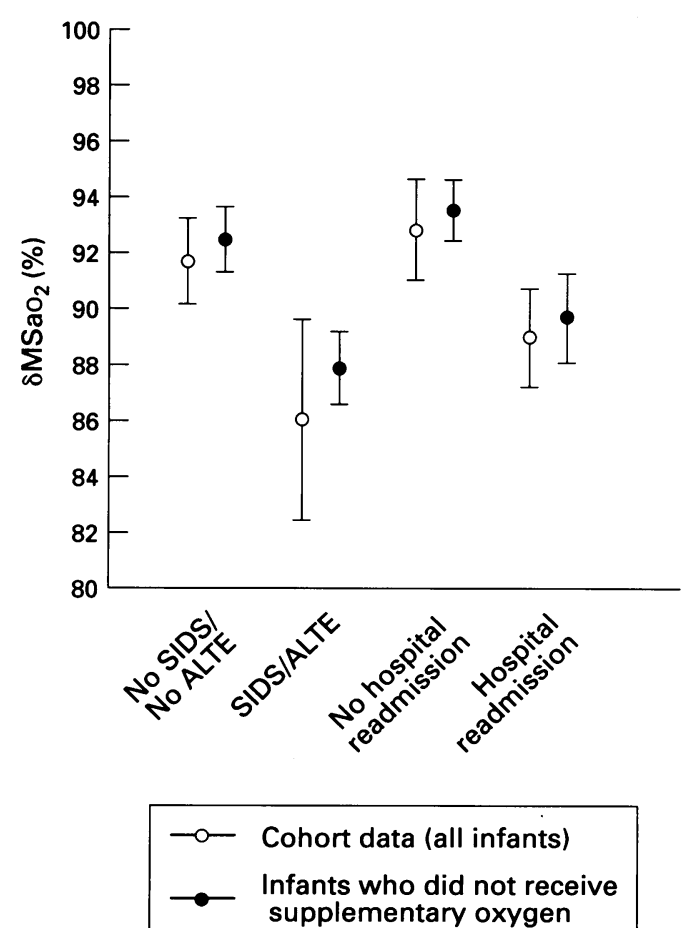

to changes in oxygen tension. ${ }^{12}$ Recently it has been shown that infants with chronic lung disease also appear to have a reduced chemoreflex response to hypoxia. This is suggested by two studies: Sekar and Duke ${ }^{13}$ reported that infants with bronchopulmonary dysplasia had lower oxygen saturation and a higher incidence of central apnoea at time of discharge than healthy preterm infants. Subsequent administration of oxygen to increase arterial saturation decreased the occurrence of central apnoea and periodic breathing in infants with chronic lung disease. Calder et $a l^{14}$ showed a markedly reduced ventilatory response to alternate breaths of air and an $\mathrm{FiO}_{2}$ of 0.16 in infants with chronic lung disease compared with preterm infants who had not had chronic lung disease, even though the former were no longer oxygen dependent at the time of the study and were well oxygenated in air. They speculated upon a blunted chemoreflex respiratory response to oxygen in infants with bronchopulmonary dysplasia which may predispose them to SIDS/ALTE and possible respiratory failure. Our data support such speculation.

In our study, the decision to discharge a patient from the neonatal unit was made by clinical assessment with additional data available from real time pulse oximetry recordings in air. Despite this assessment, $31 \%$ of the infants who were discharged from the unit without supplemental oxygen had a mean arterial saturation below $90 \%$ when the data were analysed. This finding reconfirms other published data that clinical examination is not a sensitive discriminator of hypoxaemia. ${ }^{15}$

It is often difficult to apply the pulse
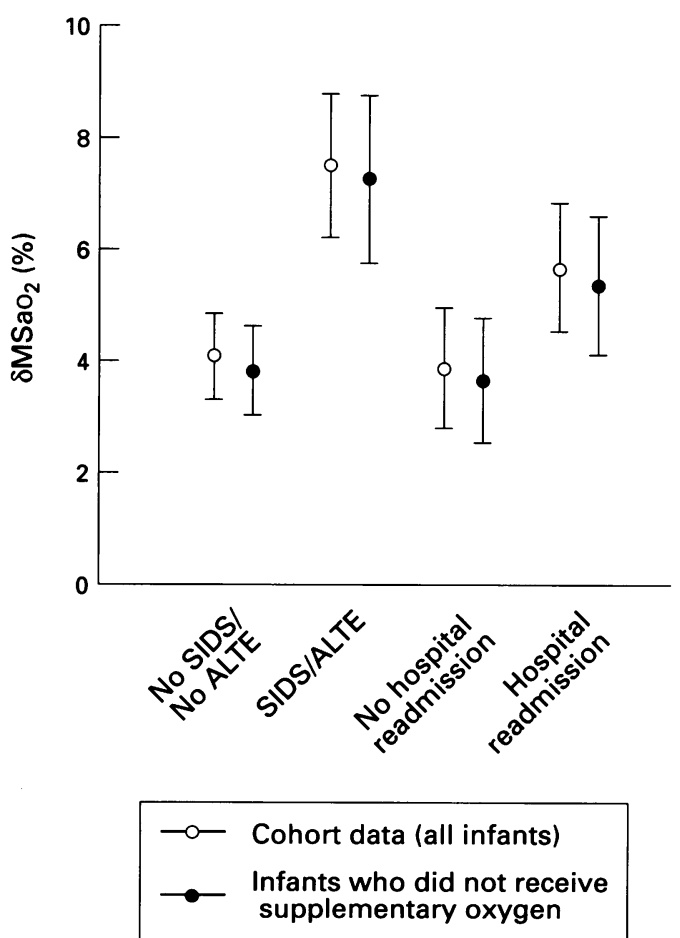

Figure $4 \quad \mathrm{SMSaO}_{2}$ and $95 \%$ confidence intervals for clinical outcome groups. SIDS/ALTE v no SIDS/no ALTE: whole cohort, $p=0 \cdot 0001$; infants not receiving supplemental oxygen, $p=0.005$. Hospital readmission $\mathrm{v}$ no hospital readmission: whole cohort, $p=0 \cdot 02$; infants not receiving supplemental oxygen, $p=0.03$. 
oximeter probe to small feet and hands. This may lead to malfunction; in most cases the malfunction causes an alarm condition, but often the device will indicate a falsely low $\mathrm{SaO}_{2}$. This is caused by what has been termed the penumbra effect ${ }^{16}$ and is due to the red and infrared light emitting diodes not being exactly mechanically incident to each other. This may cause erroneous arterial oxygen saturation recordings, which are still within the normal range. By recording two independent channels for heart rate in each infant we were able to identify periods where there was more than a $5 \%$ difference between the heart rate obtained from the heart rate monitor and that from the Ohmeda pulse oximeter. These periods could then be excluded by further analysis. Many pulse oximeters allow for time periods of up to two hours to be collected and displayed. These trend data are not supported by information from other channel recordings such as heart rate. They may therefore not be accurate. Our findings show that up to $81 \%$ of data from recordings of six hours or more may be influenced by artefact. Pulse oximeters differ in their bias, accuracy, and sensitivity. We used an Ohmeda Biox 3700 pulse oximeter which may give generally lower values for oxygen saturation than the Nellcor N-100 pulse oximeter. ${ }^{17}$ The interpretation of data from one product may not be directly transferable to another.

To lessen the possibility of non-representative periods of pulse oximetry being analysed, several hours of data should be recorded at a time and artefact excluded to give both the mean and variability. 'Spot' oximetry is often recorded in the outpatients department. Further analysis is not necessary in infants whose arterial saturation values are above $92 \%$ or below $90 \%$, when the decision to prescribe oxygen does not pose a clinical dilemma. However, in the infant whose spot recordings of arterial saturation gives values between $90 \%$ and $92 \%$, more detailed analysis is necessary before the clinical decision to continue oxygen is made.

Individual infants whose $\mathrm{MSaO}_{2}$ is accurately assessed to be below $90 \%$ can be protected from increased morbidity and even mortality by the prescription of supplemental oxygen to increase the $\mathrm{MSaO}_{2}$. The parents of infants with $\mathrm{MSaO}_{2}$ values above $90 \%$ but below the normal range should be warned to be vigilant during respiratory infections. The provision of apnoea monitors, an emergency home oxygen supply, and the training of parents in basic infant resuscitation may also be better targeted.

RI was supported by a grant from The Scottish Cot Death Trust from August 1992 to January 1995 . We would like to consultant neonatologists at the Simpson Memorial Maternity consultant neonatologists at the Simpson $M$
Pavilion, for their support during the study.

1 Bancalari E, Abdendour G, Feller R, Gannon J. Bronchopulmonary dysplasia, clinical presentation. f Pediatr 1979; 95: 819-23.

2 Tammela OK, Koivisto ME. A 1 year follow-up of low birth weight infants with and without bronchopulmonary dysplasia: health, growth, clinical lung disease, cardiovascular and neurological sequelae. Early Hum Dev 1992; 30: and neurol

3 Abman SH, Burchell MF, Schaffer MS, Rosenberg AA. Late sudden unexpected deaths in hospitalized infants with bronchopulmonary dysplasia. Am $\mathcal{F}$ Dis Child 1989; 143: 815-9.

4 Sauve RS, McMillan DD, Mitchell I, Creighton D, Hindle NW, Young L. Home oxygen therapy. Outcome of infants discharged from NICU on continuous treatment. Clin Pediatr 1989; 28: 113-8.

5 Northway WH, Rosale RC, Parker D. Pulmonary Disease following respirator therapy of Hyaline Membrane Disease. $N$ Engl f Med 1967; 267: 357-68.

6 Shennan AT, Dunn MS, Ohlsson A, Lennox K, Hoskins E. Abnormal pulmonary outcomes in premature infants: Prediction from oxygen requirement in the neonatal period. Pediatrics 1988;82: 527-32.

7 Bass C, Badger P, McIntosh N, Lafebar HN, eds. Mary 3: a new generation computer monitoring system. In: Fetal and neonatal physiological measurement. Amsterdam: Elsevier, 1991: 187-90

8 US Department of Health and Human Services. Apnoea (infantile) and home monitoring: report of a consensus development conference. Bethesda, Maryland: Public Health Service, National Institute of Health, 1986: 87.

9 Altman D, Bland J. Diagnostic tests 3: receiver operating characteristic plots. BMf 1994; 309: 188.

10 Timms RM, Khaja FU, Williams GW, and the Nocturnal Oxygen Therapy Trial Group. Haemodynamic responses to oxygen therapy in chronic obstructive pulmonary disease. Ann Intern Med 1985; 102: 29-36.

11 Baudouin SV, Waterhouse JC, Tahtamouni T, Smith JA, Baxter J, Howard P. Long term domiciliary oxygen treatment for respiratory failure reviewed. Thorax 1990; 45: ment for

12 Haliday HL, Dumpit FM, Brady JP. Effects of inspired oxygen on echocardiographic assessment of pulmonary vascular resistance and myocardial contractility bronchopulmonary dysplasia. Pediatrics 1980 ; 65: 536-40.

13 Sekar KC, Duke JC. Sleep apnoea and hypoxaemia in recently weaned premature infants with and without Bronchopulmonary dysplasia. Pediatr Pulmonol 1991; 10: $112-6$.

14 Calder C, Williams B, Smyth J, Boon A, Kumar P, Hanson $\mathrm{H}$. Absence of ventilatory responses to alternating breaths of mild hypoxia and air in infants who have had bronchopulmonary dysplasia: implications for the risk of sudden infant death. Pediatr Res 1994; 35: 677-81.

15 Wang EE, Milner RA, Navas L, Maj H. Observer agreement for respiratory signs and oximetry in infants hospitalized with lower respiratory infections. Am Rev Resp Dis 1992; 145: 106-9.

16 Kelleher JF, Ruff RH. The penumbra effect: vasomotion dependent pulse oximeter artefact due to probe malposition. Anaesthesiology 1989; 71: 787-91.

17 Thilo EH, Andersen D, Wasserstein ML, Schmidt J, Luckey D. Saturation by pulse oximetry: comparison of the results obtained by instruments of different brands. f Pediatr 1993; 122: 620-6. 